\title{
FUNCTIONS OF DIRECT INTEGRALS OF OPERATORS ${ }^{1}$
}

\author{
T. R. CHOW AND F. GILFEATHER
}

\begin{abstract}
This paper contains two results. The first one is that the unitary dilation of a direct integral of linear contraction operators is the direct integral of unitary dilations. For each linear contraction operator $T$ on a Hilbert space, consider $f(T)$ as a bounded linear operator. The second result states that if $T=\int \bigoplus T(s) d \mu(s)$ is decomposable then so is $f(T)$ and $f(T)=\int \bigoplus f(T(s)) d \mu(s)$.
\end{abstract}

In this paper all operators will be bounded linear operators on separable complex Hilbert spaces. An operator is called primary if the von Neumann algebra it generates is a factor [9]. It was first shown by J. von Neumann $[10, \S 20]$ that an arbitrary operator can be represented as a direct integral of primary operators.

In the first section of this paper we prove that the unitary dilation [5] of a direct integral of contraction operators is the direct integral of unitary dilations. For the discrete case, this result is due to Schreiber. He proved that the unitary dilation of a direct sum of contraction operators is the direct sum of the unitary dilations [6]. In the second section, we prove that if $T=\int_{\Sigma} \oplus T(s) d \mu(s)$ is a direct integral of contraction operators, then $f(T)$ defined as a bounded operator is decomposable, and $f(T)=\int_{\Sigma} \oplus f(T(s)) d \mu(s)$.

The authors wish to thank N. Suzuki for his lectures given at University of British Columbia which inspired this work.

1. The unitary dilation of a direct integral of contraction operators. The concept of direct integral or generalized direct sum of Hilbert spaces was first introduced by von Neumann [10]. This subject is now incorporated in several books, most notably, [2, Chapter II] and [8, Chapter I].

The purpose of this section is to prove that the unitary dilation of a direct integral of contraction operators on a direct integral of Hilbert spaces is the direct integral of unitary dilations. For convenience, we shall refer to [2, Chapter II] for the definitions and results concerning the direct integral of Hilbert spaces and the direct inte-

Received by the editors August 5, 1970.

AMS 1970 subject classifications. Primary 47A20, 47C15.

Key words and phrases. Direct integrals, primary operators, dilations, strong operator measures and von Neumann algebras.

1 This work was done while the first author was supported by Canadian NRC Grant 67-4058 and the second author was an Office of Naval Research Postdoctoral Associate at Indiana University.

Copyright @ 1971, American Mathematical Society 
gral of operators. For the basic properties of the unitary dilation, which we shall use, we refer to [5, Chapter I].

Let $\Sigma$ be a locally compact Hausdorff space (for our purposes a compact subset of the real line is sufficient) and $\mu$ a positive Borel measure on $\Sigma$. Let $H$ be the direct integral of Hilbert spaces

$$
H=\int_{\Sigma} \oplus H(s) d \mu(s)
$$

which satisfies [2, Definition II. 1.5.3]. Whenever there is no chance of confusion we shall surpress the $\Sigma$. An operator $A$ on $H$ is called a direct integral of operators or a decomposable operator on $H$ if $A$ $=\int \oplus A(s) d \mu(s)$ satisfies [2, Definition II. 2.3.2]. If $\psi \in H$ and $A$ is a decomposable operator on $H$, then $A \psi(s)=A(s) \psi(s)$, where $A(s)$ is an operator on $H(s)$. Let $T$ be a contraction operator on a Hilbert space $H$. The operator $U$ on the Hilbert space $K$ is a unitary dilation of $T$ if $H$ is a closed subspace of $K$ and $\left\langle T^{n} h_{1}, h_{2}\right\rangle=\left\langle U^{n} h_{1}, h_{2}\right\rangle$ whenever $h_{1}, h_{2} \in H$ and $n$ is any natural number $(\langle\cdot, \cdot\rangle$ is the inner product on $K)$. The dilation $U$ on $K$ of $T$ on $H$ is minimal if $K$ $=\operatorname{span}\left\{U^{n} h \mid n \in Z\right.$ and $\left.h \in H\right\}$.

THEOREM 1. Let $\mathcal{H}=\int \oplus H(s) d \mu(s)$ be a direct integral of Hilbert spaces and $T=\int_{\Sigma} \oplus T(s) d \mu(s)$ be a decomposable contraction on $\mathcal{H}$. Let $V(s)$ on $K^{\prime}(s)$ be a minimal unitary dilation of $T(s)$ on $H(s)$ whenever $\|T(s)\| \leqq 1$. There exists a direct integral Hilbert space $\mathfrak{K}=\int \oplus K(s) d \mu(s)$ and a decomposable unitary operator $U=\int \oplus U(s) d \mu(s)$ on $\nVdash$ such that $U$ is a minimal unitary dilation for $T, K^{\prime}(s)=K(s) \mu$-a.e. and $U(s)$ $=V(s) \mu$-a.e.

Proof. Since $T$ is a contraction, there exists a set $\delta$ of $\mu$-measure zero such that for $s \notin \delta$ we have $\|T(s)\| \leqq 1$. For $s \in \delta$ we shall define $K(s) \equiv\{0\}$ and for $s \notin E$ we shall define $K(s) \equiv K^{\prime}(s)$.

First we must show that $\mathscr{K}=\int \oplus K(s) d \mu(s)$ exists. By [2, Definition II. 1.3.1] there exists a fundamental sequence of measurable vectors $\left\{\psi_{n}\right\}$ for $\mathcal{H}$. For any integer $k$ define the functions $\zeta_{k n}$ by

$$
\begin{aligned}
\zeta_{k n}(s) & \equiv V^{k}(s) \psi_{n}(s), & & \text { if } s \notin \delta, \\
& \equiv 0, & & \text { if } s \in \delta .
\end{aligned}
$$

Now consider the set of all finite rational linear combinations of the set $\left\{\zeta_{k n}\right\}$. This latter set is denumerable and we shall denote it by $\left\{\phi_{n}\right\}$. First we claim that the scalar functions $\left\langle\phi_{n}(\cdot), \phi_{m}(\cdot)\right\rangle$ are $\mu$-measurable. To see this we need only consider the scalar function 


$$
\begin{aligned}
\left\langle\zeta_{n k}(s), \zeta_{m i}(s)\right\rangle & =\left\langle V^{n}(s) \psi_{k}(s), V^{m}(s) \psi_{i}(s)\right\rangle \quad \mu \text {-a.e. } \\
& =\left\langle V^{n-m}(s) \psi_{k}(s), \psi_{i}(s)\right\rangle \\
& =\left\langle T^{n-m}(s) \psi_{k}(s), \psi_{i}(s)\right\rangle
\end{aligned}
$$

where we use the usual convention, $T^{-|n|}(s)=T^{*|n|}(s)$. Since $T$ is decomposable, by [2, Proposition II. 2.1.1] the scalar functions $\left\langle\zeta_{n k}(\cdot), \zeta_{m i}(\cdot)\right\rangle$ are all $\mu$-measurable. Therefore the functions $\left\langle\phi_{n}(\cdot), \phi_{m}(\cdot)\right\rangle$ are all $\mu$-measurable. Next we shall show that $\left\{\phi_{n}(s)\right\}$ is dense in $K(s) \mu$-a.e. Let $s$ not belong to $\delta$. Since $V(s)$ on $K(s)$ is a minimal unitary dilation of $T(s)$ on $H(s)$, we have

$$
\operatorname{span}\left\{V^{n}(s) h \mid n \in Z, h \in H(s)\right\}=K(s) .
$$

Since $\left\{\psi_{n}(s)\right\}$ is dense in $H(s)$, it follows that,

$$
\left\{\phi_{n}(s)\right\}^{-}=\operatorname{span}\left\{\zeta_{n k}(s)\right\}=\operatorname{span}\left\{V^{n}(s) \psi_{k}(s)\right\}=K(s) .
$$

Thus the sequence $\left\{\phi_{n}\right\}$ satisfies the hypothesis of [2, Proposition II. 1.2.4] and it follows the $\mathscr{K}=\int \oplus K(s) d \mu(s)$ is defined in such a manner that $\mathfrak{H}=\int \oplus H(s) d \mu(s)$ is a closed subspace of $\mathcal{K}[2$, Proposition II. 1.7.9].

Finally we must show that $U=\int \oplus U(s) d \mu(s)$, where

$$
\begin{aligned}
U(s) & =V(s), & & \text { if } s \notin E, \\
& =0, & & \text { if } s \in E,
\end{aligned}
$$

defines a bounded linear operator which is a minimal unitary dilation of $T$. From the definition of $\left\{\phi_{n}\right\}$ it follows that the function $s$ $\rightarrow U(s) \phi_{n}(s)$ is again in the set $\left\{\phi_{n}\right\}$. Hence we have the measurability of $\left\langle U(\cdot) \phi_{n}(\cdot), \phi_{m}(\cdot)\right\rangle=\left\langle\phi_{j}(\cdot), \phi_{m}(\cdot)\right\rangle$ for some $j$. Therefore by $[2$, Proposition II. 2.1.1] it follows that $U: s \rightarrow U(s)$ is measurable. It is clear that $U$ is a unitary dilation of $T$ and the fact that $U$ is minimal also follows easily. If $\phi$ is orthogonal to $\operatorname{span}\left\{U^{n} \psi \mid n \in Z\right.$ and $\left.\psi \in \mathcal{H}\right\}$, then $\phi(s) \perp\left\{U^{n}(s) \psi_{m}(s) \mid n \in Z, m>0\right\} \mu$-a.e. Since $U(s)=V(s) \mu$-a.e., $V(s)$ is a minimal dilation of $T(s) \mu$-a.e. and $\left\{\psi_{n}(s)\right\}$ is dense in $H(s)$ $\mu$-a.e., we may conclude that $\phi(s)=0 \mu$-a.e. and $\phi=0$. This completes the proof of the theorem.

As we remarked in the introduction, whenever the measure $\mu$ is discrete the direct integral decomposition becomes the usual direct sum. If we want to consider only primary decompositions, that is, decompositions of $T$ into primary components as given by $[2, \mathrm{II}, \S 6]$, then it is not always clear when the decomposition will be discrete. However, for a large class of contractions this decomposition is always discrete. Whenever $T$ is a completely nonunitary contraction such 
that $I-T^{*} T$ is compact, then the second author has shown that $T$ is decomposed into the direct sum of primary contractions $[4$, Theorem 1]. Furthermore in this latter case each primary contraction is just a finite direct sum of an irreducible contraction having the same compactness property as the original operator $[4$, Theorem $3]$.

2. The functional calculus. As in $\$ 1$, we let $T=\int \oplus T(s) d \mu(s)$ be a decomposable contraction operator on $\mathfrak{H}=\int \oplus H(s) d \mu(s)$; and $U$, its minimal unitary dilation on $\mathcal{K}$. Let $E$ be the spectral resolution of $U$ such that $U=\int_{\Lambda(E)} \lambda d E(\lambda)$, where $\Lambda(\cdot)$ denotes the support of the measure E. B. Sz.-Nagy and C. Foiaş have defined $f(A)$ as a (not necessarily bounded) operator on $\mathcal{H C}$ for a special class of functions [5]. However, for our purpose, we shall use a definition developed by Schreiber [7]. Let $P$ be the canonical projection from $\mathcal{K}$ onto $\mathcal{H}$. For every borel subset $\alpha$ of the complex plane $C$, let $F(\alpha)=P E(\alpha) P$. Then $F$ is a positive operator-valued measure on $\mathcal{H}$ such that $\Lambda(F)$ $=\Lambda(E)=\sigma(U)$, where $\sigma(U)$ denotes the spectrum of $U[7]$. We shall call $F$ the strong operator measure of $T$. For every $F$-essentially bounded function $f, f(T)$ is an operator on $\mathfrak{H C}$ such that, for every pair of elements $x, y$ in $\mathfrak{F}$,

$$
\langle f(T) x, y\rangle=\int_{c} f(z) d\langle F(z) x, y\rangle .
$$

Proposition. Let $T=\int \oplus T(s) d \mu(s)$ be a decomposable contraction operator on $\mathcal{H}=\int \bigoplus H(s) d \mu(s)$, and $F$ be the strong operator measure of $T$. Denote by $\leftrightarrow$ the $\sigma$-field of all borel subsets of complex plane $C$. Then $F$ is decomposable and, for every $\alpha$ in $\beta, F(\alpha)=\int \oplus_{s}(\alpha) d \mu$, where $F_{s}$ is the strong operator measure of $T(s)$ whenever $\|T(s)\| \leqq 1$.

Proof. In view of the proof of Theorem 1, we may assume without the loss of generality that $\|T(s)\| \leqq 1$ for all $s$ in $\Sigma$. Since, for every $s \in \Sigma, H(s)$ is a closed subspace of $K(s)$ and $\mathfrak{H}=\int \oplus H(s) d \mu(s)$ is a closed subspace of $\mathfrak{K}=\int \bigoplus K(s) d \mu(s)$, it follows from [2, Lemma 3 , p. 189] that the canonical projection $P$ is decomposable and $P$ $=\int \oplus P(s) d \mu(s)$, where $P(s)$ is the canonical projection from $K(s)$ on to $H(s)$. It was shown by the first author that if $U$ is decomposable into a direct integral of unitary operators as $U=\int \oplus U(s) d \mu(s)$, then so is its spectral resolution $E$ [1, Theorem 2.7]. In fact, for each $\alpha \in ß, E(\alpha)=\int \bigoplus E_{s}(\alpha) d \mu(s)$, where $E_{s}(\alpha)$ is the spectral resolution of $U(s)$ for each $s$ in $\Sigma$. Thus 


$$
F(\alpha)=P E(\alpha) P=\int \bigoplus P(s) E_{8}(\alpha) P(s) d \mu(s)=\int \bigoplus F_{s}(\alpha) d \mu(s) .
$$

The proposition is proven.

Let $B(\Sigma)$ denote the $\sigma$-field of Borel subsets of $\Sigma$ and let $\delta^{c}$ denote the complement of $\delta$ in $C$. If $A=\int \oplus A(s) d \mu(s)$ is a scalar operator in Dunford's sense [3], then the spectrum $\sigma(A)$ of $A$ is

$$
\cap\left\{\left\{\bigcup_{\sigma}(A(s)) \mid s \in \delta, \delta \in \mathbb{B}(\Sigma)\right\}-\mid \mu\left(\delta^{c}\right)=0\right\}
$$

[1, Lemma 2.6]. Since $\Lambda\left(F_{s}\right)=\Lambda\left(E_{8}\right)=\sigma(U(s))$, it follows that

$$
\mathfrak{L}_{\infty}(F)=\bigcup\left\{\left\{\cap \mathscr{L}_{\infty}\left(F_{s}\right) \mid s \in \delta, \delta \in B(\Sigma)\right\} \mid \mu\left(\delta^{c}\right)=0\right\} .
$$

$\left(\mathscr{L}_{\infty}(\cdot)\right.$ denotes the vector space of essentially bounded scalar-valued functions.)

TheOREM 2. Let $T=\int \oplus T(s) d \mu(s)$ be a decomposable contraction operator on $\mathfrak{H}=\int \bigoplus H(s) d \mu(s)$; and $F$, the strong operator measure of $T$. If $f$ is an F-essentially bounded function, then $f(T)$ is decomposable and $f(T)=\int \bigoplus f(T(s)) d \mu(s)$ with $f(T(s)$ ) well-defined $\mu$-a.e. Conversely, if $f$ is $F_{s}$-essentially bounded for $\mu$-a.a.s, then there is a $\mu$ measurable vector field of operators $s \rightarrow g(T)(s)$ ) such that $g(T(s))$ $=f(T(s)) \mu$-a.e. and $f(T)=\int \bigoplus g(T(s)) d \mu(s)$.

Proof. Suppose that $f \in \mathscr{L}_{\infty}(F)$. Then it follows from the proposition and Fubini's theorem that, for any arbitrary pair of elements $x$ and $y$ in $\mathfrak{H C}$,

$$
\begin{aligned}
\langle f(T) x, y\rangle & =\int_{\mathbf{C}} f(z) d\langle F(z) x, y\rangle=\int_{\mathbf{C}} f(z) \int_{\Sigma} d\left(F_{s}(z) x(s), y(s)\right) d \mu(s) \\
& =\int_{\Sigma} \int_{\mathbf{C}} f(z) d\left\langle F_{s}(z) x(s), y(s)\right\rangle d \mu(s) \\
& =\left\langle\left[\int_{\Sigma} \bigoplus f(T(s)) d \mu(s)\right] x, y\right\rangle .
\end{aligned}
$$

This proves the first part of the theorem. Conversely, if $f$ is $F_{\mathrm{s}}$ essentially bounded for $\mu$-a.a.s., then as remarked previously, $f$ is $F$-essentially bounded so that $f(T)$ is well-defined. Let $\delta$ be a subset of $\Sigma$ of measure zero such that, for $s \notin \delta, f$ is $F_{s}$-essentially bounded. Let $s \rightarrow g(T(s))$ be such that $g(T(s))=f(T(s))$ for $s \notin \delta$ and zero otherwise. It is clear that $s \rightarrow g(T(s))$ is $\mu$-measurable. A similar argument as in the proof of the first part of the theorem provides the decomposability of $f(T)$ as well as the representation $f(T)=\int \bigoplus g(T(s)) d \mu(s)$. 


\section{REFERENCES}

1. T. R. Chow, A spectral theory for the direct integral of operators, Math Ann. 188 (1970), 285-303.

2. J. Dixmier, Les algèbres d'opérateurs dans l'espace hilbertien, Cahiers Scientifiques, fasc. 25, Gauthier-Villars, Paris, 1957. MR 20 \#1234.

3. N. Dunford, $A$ survey of the theory of spectral operators, Bull. Amer. Math. Soc. 64 (1958), 217-274. MR 21 \#3616.

4. F. Gilfeather, The structure theory of N. Suzuki for non-selfadjoint operators in Hilbert spaces (to appear).

5. B. Sz.-Nagy and C. Foiaş, Analyse harmonique des operateurs de l'espace de Hilbert, Masson, Paris; Akad. Kiad6, Budapest, 1967. MR 37 \#778.

6. M. Schreiber, Unitary dilations of operators, Duke Math. J. 23 (1956), 579-594. MR 18, 748.

7. - A functional calculus for general operators in Hilbert spaces, Trans. Amer. Math. Soc. 87 (1958), 108-118. MR 20 \#6040. $\# 547$.

8. J. T. Schwartz, $W^{*}$-algebras, Gordon and Breach, New York, 1967. MR 38

9. N. Suzuki, The algebraic structure of non-selfadjoint operators, Acta Sci. Math. 27 (1966), 173-184. MR 35 \#5971.

10. J. von Neumann, On rings of operators. Reduction theory, Ann. of Math. (2) 50 (1949), 401-485. MR 10, 548.

Oregon State University, Corvallis, Oregon 97331

University of British Columbia, Vancouver 8, British Columbia, Canada

University of Hawali, Honolulu, Hawail 96822 